\title{
Forcing, Freedom, \& Uniqueness in Graph Theory \& Chemistry
}

\author{
Douglas J. Klein* and Vladimir Rosenfeld
}

Texas A\&M University at Galveston, Galveston, TX 77553

Abstract. Harary's \& Randić's ideas of "forcing" \& "freedom" involve subsets of double bonds of Kekule structure such as to be unique to that Kekule structure. Such forcing sets are argued to be greatly generalizable to deal with various other coverings, and thence forcing seems to be fundamental, and of notable potential utility. Various forcing invariants associated to (molecular) graphs ensue, with illustrative (chemical) examples and some mathematical consequences being provided. A complementary "uniqueness" idea is noted, and the general characteristic of "derivativity" of "forcing" is established (as is relevant for QSPR fittings). Different ways in which different sorts of forcings arise in chemistry are briefly indicated.(doi: 10.5562/cca2000)

Keywords: forcing, freedom, Kekule structures, Clar structures, Pauling bond orders, conjugated circuits, resonance structures, maximum matchings, maximal matchings, radicals

\section{HYSTORY \& MOTIVATION}

Professor Frank Harary was arguably "the" leading popularizer of graph theory, as in his classic influential text ${ }^{1}$ (along with perhaps a half dozen other co-authored monographs), while also he was the most prolific researcher in the field (in terms of published articles), and yet further he was intently interested in whatever science utilized graph theory - even chemistry, having published therein several articles, including a prominent position in Balaban's early edited monograph ${ }^{2}$ on Chemical Applications of Graph Theory. Thus Frank (as Professor Harary preferred to be called by friends) was a special invited speaker at the 1991 mathematical chemistry conference in Galveston, where there was to be a strong showing of "chemical graph theory". Further, Frank was invited to submit a manuscript for consideration for publication in a special edition ${ }^{3}$ of $\mathrm{J}$. Math. Chem. devoted to this meeting, and the day of the third \& final dead-line for submission of such manuscripts, he submitted (via FAX) four pages of handscrawled notes (broadly concerning benzenoids) for consideration for such a paper. These notes were too far from being a completed paper to be included in the special issue. Indeed a good portion of Frank's notes presented various ideas, results, or questions, several already known in chemistry - though now approached from a more purely graph-theoretic more general viewpoint. One of these ideas was that of "forcing" ex- pressed in the context of Kekule structures, or "perfect matchings": a set of double bonds of a given Kekule structure is said to be forcing if there is no other Kekule structure containing the same set of double bonds. But this and particularly his idea of "forcing number" of a Kekule structure (as the minimum number of such forcing bonds) had already been conceived by Milan Randić under the title of "degree of freedom" of a Kekule structure, with two papers indicating some chemical relevance already published. ${ }^{4,5}$ Frank argued that the idea of "forcing" was much more general, and that the "forcing" nomenclature was better. He encouraged collaboration on the topic, which indeed ensued: a degree of concurrence of opinions was achieved, some initial results were established (on forcing, and some other ideas), and a paper subsequently appeared. ${ }^{6}$

Following this work, forcing for Kekule structures has continued to receive attention, in both the mathematical, ${ }^{7-18}$ and the chemical ${ }^{19-27}$ literature. And further, what has been termed an "anti-forcing" idea has also been proposed ${ }^{17} \&$ studied. ${ }^{17,28-33}$ All this would surely immensely please Frank - including the evident efficacy of his nomenclature, such as deeply interested him, in a very general context.

Now perhaps is a good time to emphasize the wider generality of the "forcing" idea, as is attempted here, indicating that "anti-forcing" is part of this broad general picture, with further illustration of the extended idea, though first retaining the context of Kekule struc-

\footnotetext{
* Author to whom correspondence should be addressed. (E-mail: kleind@tamug.edu)
} 
tures. But after this initial discussion, the idea is further generalized to deal with maximum matchings, maximum Clar structures, so-called " $A[B]$-coverings", and on to " $A \mid B$-coverings", and then yet further, ultimately beyond even the realm of graph theory. Relevances for chemical resonance-related ideas are noted, both from the point of view of Pauling \& of Clar, and also possible relevance is noted for statistical mechanical modelling of locally structured fluids. It is suggested that whenever such globally spanning graphic structures are chemically important, then also associated mathematically fundamental ideas of forcing via "local substructures" should be of chemical relevance. That is, in chemistry, properties are often viewed to be appear in some local way, with "forcing" one generally means by which to manifest the "local" in terms of the "global" (e.g., Kekule structures). The interconnection of "local" to "global" is also here described in terms of a complementary idea of "uniqueness" (which might be viewed as an "anti-analogue" of forcing). Some few consequent results for forcing invariants are mentioned, particularly their "derivativity" and their mode of use in describing molecular properties which are commonly thought of as "additive".

\section{FORCING FOR KEKULE STRUCTURES}

Here, fairly standard graph theoretical nomenclature and notation form the background, say following Ref. 1 or Ref. 34. Thus $G$ denotes a graph with vertex set $V(G)$ and edge set $E(G)$. A subgraph $H$ of $G$ is such that $V(H) \subseteq V(G)$ \& $E(H) \subseteq E(G)$. A spanning subgraph of $G$ is one such that $V(H)=V(G)$. The $G$-complement $H^{c G}$ of a subgraph $H$ of $G$ is that which has $E\left(H^{c G}\right)=\{\{i, j\} \in E(G): i, j \in V(G),\{i, j\} \notin E(H)\}$ and $V\left(H^{c G}\right) \equiv e_{e}^{\in E\left(H^{c G}\right)} e$. A perfect matching - or more chemically, a (fully neighbor-paired) Kekule structureof $G$ is a spanning subgraph $\kappa$ of $G$ such that the degree of every site of $\kappa$ is 1 . In modelling absorption of dimers on surfaces, these Kekule structures are often termed "dimer coverings". The number of Kekule structures of $G$ is denoted $K(G)$, and graphs with $K(G) \geq 1$ are called Kekulean. For a Kekule structure $\kappa$ of $G$, a cycle $C$ of $G$ is said to be conjugated if alternate edges of $C$ are in $\kappa$ (and in mathematics a conjugated cycle is often termed an "alternating cycle"). The Pauling bond order $\langle e\rangle$ of an edge $e$ of a Kekulean $G$ is the fraction of Kekule structures which contain $e$. The conjugated 6-circuit expectation $\langle C\rangle$ for a given 6cycle $C \subseteq G$ is the fraction of Kekule structures for which $C$ is conjugated. It is realized that $\langle e\rangle$ is chemi- cally relevant in Pauling's work, ${ }^{35,36}$ and $\langle C\rangle$ is relevant in "conjugated-circuits" theory. ${ }^{37-43}$

Next, our initial view of "forcing" is indicated. Given a Kekule structure $\kappa$ of a Kekulean $G$, a subset $S_{e}$ of its edges is e-forcing if no other Kekule structure contains $S_{e}$ as a subset of its edges. Also a subset $S_{(e)}$ of edges of $\kappa^{c G}$ is $(e)$-forcing if $S_{(e)}$ is contained in no $G$-complement of any other Kekule structure of $G$. For a Kekule structure $\kappa$ of $G$, a subset $S_{6}$ of conjugated 6cycles of $G$ is 6 -forcing if no other Kekule structure $\kappa$ of $G$ manifests the same subset of conjugated 6-cycles with the same conjugation pattern within each of these cycles. For each of the sets $S_{\tau}=S_{e}, S_{(e)}$, or $S_{6}$ with $\tau=e,(e)$, or 6 , define the $\tau$-freedom of $\kappa$ as the minimum order of a $\tau$-forcing of $\kappa$. The sum of $\tau$ freedoms for the Kekule structures of $G$ gives a net $\tau$ force $f_{\tau}(G)$, which evidently is a graph invariant, with $f_{\tau}(G) / K(G)$ the mean $\tau$-freedom of $G$.

An example is provided by the phenanthrene graph, of 3 hexagonal rings of which two are fused to next neighbor sides of the central ring. There are $5 \mathrm{Ke}$ kule structures, as indicated in Figure 1, with minimal $e$ forcing, (e)-forcing, \& 6-forcing sets also there indicated for each Kekule structure, and the resultant $e-,(e)-$, $\&$ 6-freedoms are noted, along with the net forces. There is seen to be a degree of correlation amongst these quantities, and it is natural to speculate as to the general extent of this. Evidently these forcings correspond to much smaller (more "local") graphs than the Kekule structures - so that one could also view them to be abbreviated representations of the various Kekule structures.

Here $e$-force corresponds to what has previously been termed simply "net forcing number" (or "net degree of freedom"), and (e)-force corresponds to what has previously been called the "net anti-forcing number". Further examples of $e$-freedoms $\& e$-forces are found elsewhere ${ }^{4,5,8-10}$ (under this earlier alternate nomenclature), and also similarly for (e)-forcing., $18,20,21$

Now Pauling bond orders can be related to $e$ forcing:

Proposition 1 - For a graph $\mathrm{G}$, let $n_{M}$ be the number of edges taking the maximum value $\mathrm{M}$ of $\langle e\rangle \cdot K(G)$, and let $\mathrm{n}_{1}$ be the number of edges for which $\langle e\rangle \cdot K(G)=1$. Then

$$
(M-1) \cdot K(G)+n_{M} \geq f_{e}(G) \geq 2 \cdot K(G)-n_{1}
$$

Proof: Clearly, to distinguish any Kekule structure $\kappa$ which shares one of the $n_{M}$ maximally shared bonds with $M-1$ other Kekule structures, one need choose no more than $M$ bonds from $\kappa$ - and at the same time the remaining at least $K(G)-n_{M}$ Kekule structures require fewer bonds, i.e., at the most $M-1$ bonds. Thus $M \cdot n_{M}+(M-1) \cdot\left(K(G)-n_{M}\right)$ is an upper bound to 


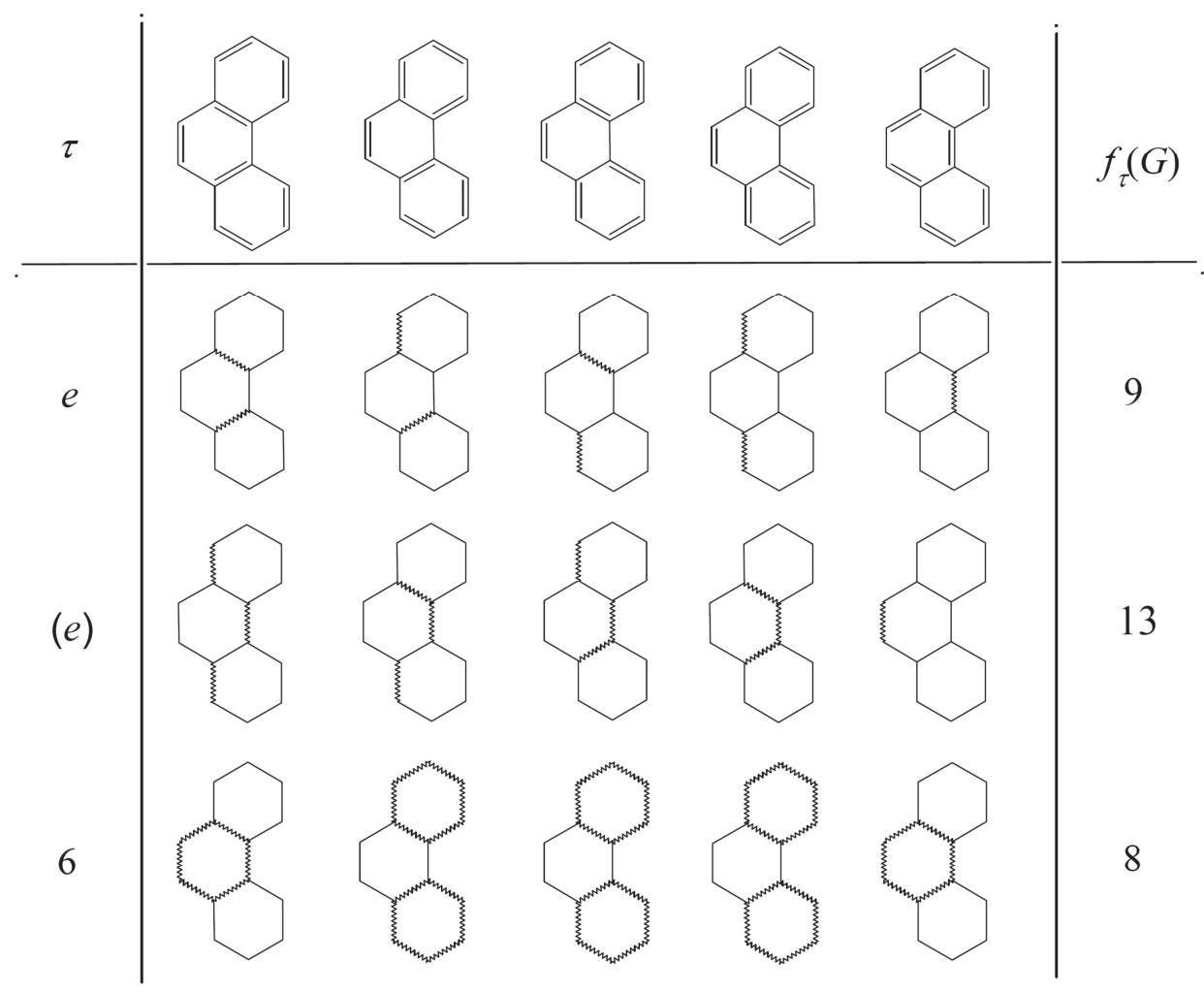

Figure 1. The 5 Kekule structures of phenanthrene, with $e$-forcing, (e)-forcing, and 6-forcing sets marked in "wiggly bold" in the respective associated graphs.

$f_{e}(G)$, as in the proposition. For the lower bound, one notes that each Kekule structure $\kappa$ requires at least 1 bond to distinguish it from the others, and the only ones which can do this are ones which give $\langle e\rangle K(G)=1$, there clearly being no more than $n_{1}$ such Kekule structures - while at least $K(G)-n_{1}$ Kekule structures require at least 2 bonds to distinguish them. Thus $n_{1}+2\left\{K(G)-n_{1}\right\}$ is a lower bound to $f_{e}(G)$, as in the proposition.

Presumably there is a similar relation of 6-forcing to conjugated circuits expectations $\langle C\rangle$. But there are some other initial questions about 6 -forcing.

\section{Admissability of 6-Forcing \& e6-Forcing}

The present definition of 6-forcing indicates a wider implementation of the forcing idea than exhibited in earlier papers. ${ }^{6-20}$ But it should be mentioned that not all Kekule structures of general graphs admit a 6-forcing set, and consequently do not admit a 6-freedom. Such clearly is the circumstance with any multi-Kekulean graph without any 6-cycles - e.g., the 4-cycle (butadiene). It is a fundamental conjecture that every Kekule structure of a Kekulean benzenoid admits a 6-forcing a benzenoid being a subgraph of the honeycomb network enclosed in a Jordan curve consisting of the outer edges of the subgraph. That is, just having every edge in a 6-cycle does not of itself guarantee the admissibility of 6-forcing - as is illustrated with the Kekule structure $\kappa$ in Figure 2 (such as is a "coronoid" - which does not meet the technical definition of a 'benzenoid").

One might imagine that our 6-forcing is admissible at least for suitable benzenoids, such as the catacondensed benzenoids which are those benzenoids with every vertex on the outer boundary. Indeed, we have:

Proposition 2 - For finite catacondensed benzenoids, every Kekule structure is 6-forced.

Proof: The simplest catacondensed species (ben-

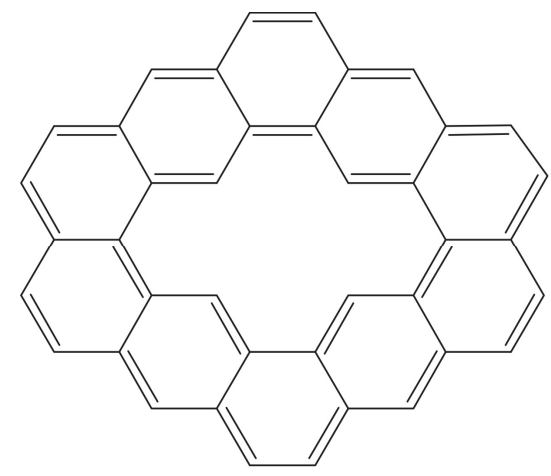

Figure 2. A coronoid structure, showing a Kekule structure without any conjugated 6-circuits. 


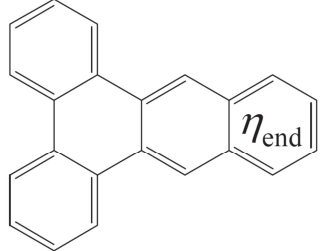

(a)

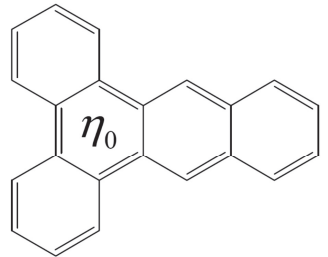

(b)
Figure 3. In (a) a terminal hexagon $\eta_{\text {end }}$ which turns out to be conjugated (in $\kappa$ ), while in (b), $\eta_{\text {end }}$ is not conjugated, though the related ring $\eta_{0}$ must be.

zene) has two Kekule structures, and with the recursive addition of hexagons, one sees that the number only increases with each addition. Thus the catacondensed benzenoids are Kekulean (as has long been known), with multiple Kekule structures. Consider a Kekule structure $\kappa$ and suppose that a (possibly empty) set $S_{\text {list }}$ of 6cycles conjugated in $\kappa$ has been selected but fails to distinguish $\kappa$ from another Kekule structure $\kappa^{\prime}$. Then $\kappa \cup \kappa^{\prime}$ contains a cycle $C$, which must be conjugated, and also forms the boundary to a catacondensed species $C^{+}$which has two Kekule structures $\kappa \cap C \equiv \kappa_{C}$ \& $\kappa^{\prime} \cap C \equiv \kappa_{C}^{\prime}$ conjugated around the boundary of $C^{+}$.

Now for such a $C^{+}$with such $\kappa_{C} \& \kappa_{C}^{\prime}$ we wish to show that $\kappa_{C}$ contains a conjugated 6-circuit. If $C^{+}$ is a 6-cycle, the result is trivially immediate. If $C^{+}$is not a 6-cycle, then consider a terminal hexagon $\eta_{\text {end }}$ of $C^{+}$(i.e., a hexagon fused to no more than one other hexagon), whence if there is conjugation around $\eta_{\text {end }}$, as in Figure 3(a), then the result is again immediate. If not, then follow from $\eta_{\text {end }}$ to the adjacent hexagon, and if this hexagon is fused to only one further on the opposite side from $\eta_{\text {end }}$, repeat continuing on to ever more distant hexagons till one $\eta_{0}$ is found without a further hexagon fused on the opposite side. This is illustrated in Figure $3(\mathrm{~b})$, where it is seen that $\eta_{0}$ must be a conjugated 6-circuit in $\kappa_{C}$.

Granted the 6-circuit in $\kappa_{C} \subseteq \kappa$, one adds this 6circuit to the list $S_{\text {list }}$, thereby distinguishing $\kappa$ from $\kappa^{\prime}$. And one similarly continues to expand $S_{\text {list }}$ till $\kappa$ is distinguished from every other Kekule structure, where $S_{\text {list }}$ has become a 6-forcing set, and our proof is completed.

One may note that the condition of finiteness is required in the proof, and in the theorem. For instance, the infinite polyacene (with no terminal hexagonal ring) has just 4 particular Kekule structures with continual alternation down each (disjoint) boundary of the strip and these 4 have no conjugated 6-circuit.
A further related definition considers subsets $S_{e 6}$ with members which are either edges or 6-cycles of $G$, so that $S_{e 6}$ is partitioned into a set $S_{e}$ of edges of $G$ and a set $S_{6}$ of 6-cycles of $G$. Then $S_{e 6}=S_{e} \oplus S_{6}$ is e6forcing for a Kekule structure $\kappa$ of $G$ if $S_{e} \subseteq E(\kappa)$, each member of $S_{6}$ is conjugated in $\kappa$, and no other Kekule structure has this same relation to $S_{e 6}$. The e6forcing number of $\kappa$ of $G$ is the minimum size of such an $S_{e 6}$. This amends the complicit restriction on the completeness of 6-forcing, as noted before proposition $\mathbf{2}$.

\section{Forcing for $A[B]$-Covers}

Actually the "forcing" concept is somewhat more general than indicated in the preceding two sections. Consider a (perhaps small) nonempty set $\mathcal{F}$ of connected isomorphicly distinct graphs and define an $\mathcal{F}$-cover of $G$ to be a spanning subgraph of $G$ such that every component is isomorphic to a member of $\mathcal{F}$. Now granted a disjoint union $\mathcal{F}=A \cup B$ with each member of $A$ having a $B$-covering (and no members of $B$ with an $A$-cover), there is amongst all the $\mathcal{F}$-covers some which have a maximum number of components isomorphic to members of $A$. Each such $A$-maximum $\mathcal{F}$ cover is termed an $A[B]$-cover of $G$. Let $\mathcal{F}_{G}$ be the set of subgraphs of $G$ such that each member of $\mathcal{F}_{G}$ is isomorphic to a member of $\mathcal{F}$. Now in correspondence to selected $F \subseteq \mathcal{F}$, we entertain a certain type of partial ordering relation ${ }_{-F}$ to select $F_{G} \in \mathcal{F}_{G}$ from within an $A[B]$-cover $\xi$ such that $F_{G}$ is isomorphic to $F \in \mathcal{F}$ there being different possible choices for the relation $-F$, say:

- $F_{G-F} \xi$ could denote just the ordinary subgraph relation $F_{G} \subseteq \xi$; or

- $F_{G-F} \xi$ could require that $F_{G}$ is a component of $\xi$ and also is induced in $G$; or

- ${ }_{-F}$ could indicate the $G$-complement relation $\bar{F}_{G} \subseteq_{c} \xi$, by which we mean that $F_{G} \subseteq \xi^{c G}$; or

- $F_{G}-F$ could mean that $F_{G} \subseteq \xi$ while also $F_{G}$ induces in $G$ to a graph containing a subgraph isomorphic to a given graph $C$ (say a cycle), whence we also could more precisely write $F_{G} \subseteq_{F, C} \xi$.

For a given choice of relation, if $F_{G-F} \xi$, we say $\xi$ is $\mathcal{F}$-consistent. If there is a unique $A[B]$-cover $\xi$ which is $\mathcal{F}$-consistent, then $\mathcal{F}_{G}$ is said to $\mathcal{F}$-force $\xi$. The minimum size of such a unique $\mathcal{F}_{G}$ is said to be the $\mathcal{F}$ freedom for $\xi$. There is an associated $\mathcal{F}$-force $f_{\mathcal{F}_{A[B]}}(G)$, which is the sum of the $\mathcal{F}$-freedom for each $A[B]$-cover $\xi$. That the relations ${ }_{-F}$ are in correspondence with the $F \in \mathcal{F}$ means that we have a set $\mathcal{R} \equiv\left\{{ }_{-F}: F \in \mathcal{F}\right\}$, so that one should generally speak of an $\mathcal{F}, \mathcal{R}$-forcing, $\mathcal{F}, \mathcal{R}$-freedoms, and an $\mathcal{F}, \mathcal{R}$-force but typically we presume the role of $\mathcal{R}$ to be understood. 
All the ideas of the preceding two sections are now recovered as special cases, mostly when a Kekule structure is identified as an $A[B]$-cover with $A=K_{2}$ (the 2-site 1-edge graph) and $B=\varnothing$ (the empty graph). Then $\mathcal{F}=\left\{K_{2}\right\}$ and when the relation ${ }_{-F}$ is $\subseteq, \mathcal{F}$ forcing becomes what we called $e$-forcing. For the relation $\subseteq_{c}, \mathcal{F}$-forcing becomes $(e)$-forcing. For the relation ${ }_{C}$ with $F=C$ being the 6-cycle, $\mathcal{F}$-forcing becomes 6-forcing. If we take $\mathcal{F}=\left\{C_{6}, K_{2}\right\}=A$, we are led to $e 6$-forcing.

Moreover, the present formulation extends beyond the ideas of the preceding two sections. A maximum matching is an $A[B]$-cover with $A=K_{2}$ (the 2-site 1edge graph) and $B=\left\{K_{1}\right\}$ (the 1-site graph). From the chemical valence-bond (VB) point of view these maximum matchings represent VB resonance structures with the possibility of unpaired electrons, though of a minimum number, and they are of especial interest when there are no fully neighbor-paired Kekule structures. One may consider $\mathcal{F}=\left\{K_{2}\right\}$ or $\mathcal{F}=\left\{K_{1}\right\}$, with associated relation $\subseteq$, to obtain two natural types of $\mathcal{F}$. forcing. Dealing with maximum matchings (instead of just simple matchings) is chemically meaningful within a resonance-theoretic context, since with the loss of each possible edge in a matching the (VB-theoretic) energy cost goes up (and the associated contribution to stability goes down). Mathematically it is more difficult (in terms of the requisite size of the forcing set $S$ ) to $e$ force a general matching, in-as-much as each potential edge would also be a potential pair of isolated sites that is, a forcing set would need to contain every edge and every adjacent pair of sites in a matching being forced.

Another nice example of $A[B]$-covers is provided by what are frequently termed ${ }^{44-49}$ "Clar structures". In this context, $A=C_{6}$ the 6-cycle $\& B=K_{2}$, and one may consider $\mathcal{F}=\left\{C_{6}\right\}, \mathcal{F}=\left\{K_{2}\right\}$, or $\mathcal{F}=\left\{C_{6}, K_{2}\right\}$ with associated relation $\subseteq$, to obtain three natural types of $\mathcal{F}$-forcing. These ideas are returned to in the next section, with a different (related) definition of Clar structure.

\section{Forcing for $A \mid B$-Covers}

Another chemically motivated slightly more generalized possibility for covers \& associated forcings deals with $A$-maximal $\mathcal{F}$-covers, by which we mean those $\mathcal{F}$ covers $\xi$ for which there are no other $\mathcal{F}=A \cup B$ covers $\zeta$ with the set of $A$-components of $\xi$ being a proper subset of those of $\zeta$. With the notation that $A_{\xi}$ is the set of $A$-components of $\xi$, then $\xi$ is $A$-maximal when there is no other $\mathcal{F}=A \cup B$-cover with $A_{\xi} \subset A_{\zeta}$ - and such maximal $\mathcal{F}=A \cup B$-covers are here denoted as $A \mid B$-covers. It is understood that for $K_{2} \mid K_{1}$ covers, there are naturally associated $\tau$-forcings, $\tau$ freedoms, and $\tau$-forces, for $\tau=e,(e), 6, \& e 6$.
Of particular chemical relevance in this regard are the maximal matchings, for which our preceding definition means those matchings $\xi$ with no adjacent sites both of which are unpaired. That is, these maximal matchings are those $\xi$ for which the subset of edges of $\xi$ is $\left\{K_{2}\right\}$-forcing. Chemically these unpaired sites generally cost some additional energy when they have less than the maximum number of neighbor pairings, but if there are vastly more of the non-maximum resonance patterns (as when the maximum ones are more easily $\left\{K_{2}\right\}$ - or $\left\{K_{1}\right\}$-forced), then the maximal matchings still may play a major role because of the then more significant "resonance" energy. Indeed, maximal matching resonance structures are often mentioned by Pauling, e.g., in his master work of Ref. 24. Notably especially for large benzenoids the molecular boundaries might ${ }^{50,51}$ severely constrain the Kekule structures, or even maximum matchings, whence the maximal ones can become not just contributory but crucial, and dominant. Indeed this recognition has enabled ${ }^{38,39,52,53}$ general simple (chemical) results in understanding defects in graphene that is, for the occurrence of unpaired electrons localized near boundaries, vacancy defects, and more.

Also maximal matchings naturally occur in the consideration (e.g., as in Refs. 54 and 55) of monomerdimer coverings. Here if each dimer is permanently absorbed at neighbor pairs of positions on the lattice graph of a surface, then the process continues to a maximal extent where no two adjacent sites unoccupied by a dimer remain - that is, maximal matchings result.

As another example of an $A \mid B$-cover, consider the definition of a Clar structure as an $A \mid B$-covering with $A=C_{6} \& B=K_{2}$. These are of relevance in Clar's view $^{56}$ of electronic structure of benzenoids, and some related conjugated carbon networks. Here one may consider $\mathcal{F}=\left\{C_{6}\right\}$, or $\mathcal{F}=\left\{K_{2}\right\}$, or $\mathcal{F}=\left\{C_{6}, K_{2}\right\}$ with associated relation $\subseteq$, to obtain three natural types of $\mathcal{F}$-forcing. An example of the 4-hexagon chrysene graph is illustrated in Figure 4, showing different forcing sets for different choices of $\mathcal{F}=\left\{C_{6}\right\}$ and $\mathcal{R}$. Much as with Kekule structures, there seems to be a correlation amongst our first considered $\tau$-forces. It also seems likely that there is an intimate relation between 6-forcing (or $\left\{C_{6}\right\}$-forcing) of Kekule structures, and $\left\{C_{6}\right\}$-forcing of Clar structures. Frequently in place of $C_{6} \mid K_{2}$-covers, the smaller set of a $C_{6}\left[K_{2}\right]$-cover is considered $^{32}$ (and taken as the definition of a Clar structure). But much as for unpairing in Kekule structures, the less restrictive $C_{6} \mid K_{2}$-covers (i.e., those without a maximum number of $A=C_{6}$ components) should be crucially relevant when there are many more of them than the $C_{6}\left[K_{2}\right]$-covers.

We may note:

Observation 3 - The $\tau$-forcings of $A[B]$-coverings with $A \supseteq B$ form a partially ordered set (or poset), 


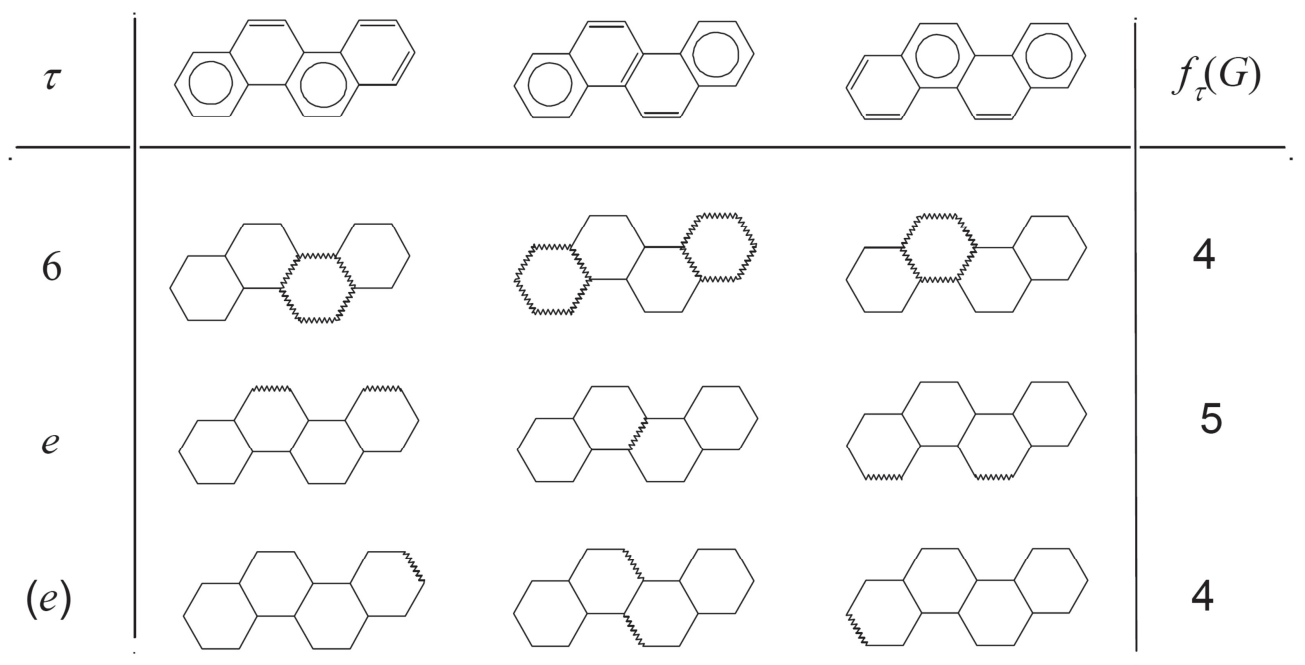

Figure 4. The 3 Clar structures of chrysene, with $e$-forcing, $(e)$-forcing, and 6-forcing sets marked in bold in the respective associated graphs.

under the subgraph partial ordering, and the poset is closed under set union.

The same is true for $A \mid B$-coverings with $A \supseteq B$.

This then provides yet another example of posets ${ }^{57}$ appearing in a chemical context.

\section{Complement to Forcing}

An idea developed by Golumbic et al. ${ }^{58}$ may be modified and extended so as to relate to $A[B]$-covering \& associated forcings. Given a subset $S$ of vertices of a graph $G$, the induced graph $S^{G}$ is that with $V\left(S^{G}\right) \equiv S$ \& $E\left(S^{G}\right) \equiv\{\{i, j\} \in E(G): i, j \in S\}$. For a subgraph $H \subseteq G$, abbreviate $V(H)^{G}$ to $H^{G}$, which is then called the subgraph induced from $H$. Thus $\left(H^{G}\right)^{G}=H^{G}$. And a subgraph $H \subseteq G$ is itself induced if $H^{G}=H$. Given an $A[B]$-covering $\xi$ of $G$, a subgraph $\xi_{A} \subseteq \xi$ composed solely from $A$-components of $\xi$ induces a subgraph $\xi_{A}{ }^{G}$ which may have but a single $A$-covering, in which case we say $\xi_{A}{ }^{G}$ is $A$-unique. Then the $A[B]$ -uniqueness $v_{A[B]}(\xi)$ is the maximum number of $A$ components in such an $A$-unique subgraph $\xi_{A}{ }^{G}$ arising from $\xi_{A} \subseteq \xi$. Define the $A[B]$-richness invariant $r_{A[B]}(G)$ for graph $G$ as the sum of $v_{A[B]}(\xi)$ over all $A[B]$-coverings of $G$. Then the mean- $A[B]$ - uniqueness $\langle v\rangle_{A[B], G}$ is $r_{A[B]}(G) / \#_{A[B]}(G)$. Given an $A[B]$-covering $\xi$ of $G$, define its $A$-order as the number $|\xi|_{A}$ of its $A$ components. Then if $\xi$ is $A$-perfect, one has $|\xi|_{A}=|V(\xi)| /|V(A)|$. Similar definitions apply for $A \mid B$-coverings.
As an example consider the $K_{2}\left[K_{1}\right]$-coverings of perinapthyl (with 3 hexagons mutually edge-fused at a single site common to all 3 ). In Figure 5 a representative set of $K_{2}\left[K_{1}\right]$-coverings $\xi$ is shown, along with a maximal $K_{2}$-unique subset for each of these coverings (with $\xi_{K_{2}}$ being understood to be the subset of edges there

$$
\xi \quad \xi_{A} \quad v_{K_{2}\left[K_{1}\right]}(\xi) \quad|\mathcal{O}(\xi)|
$$<smiles>C1=Cc2cccc3cccc(c23)C=C1</smiles>

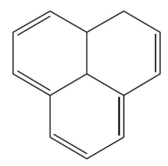<smiles>C1=Cc2cccc3c2C=CC(=C1)C3</smiles>

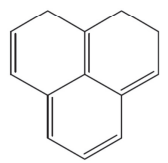<smiles>C1=Cc2cccc3c2C=CC(=C1)C3</smiles>

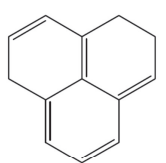

6<smiles></smiles>

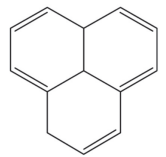

5 6

$$
r_{A[B]}(G)=5 \cdot 2+5 \cdot 6+5 \cdot 6+5 \cdot 6
$$

Figure 5. Four resonance structures of perinaphthyl, with $K_{2}$ unique subgraphs, and associated uniqueness numbers. 


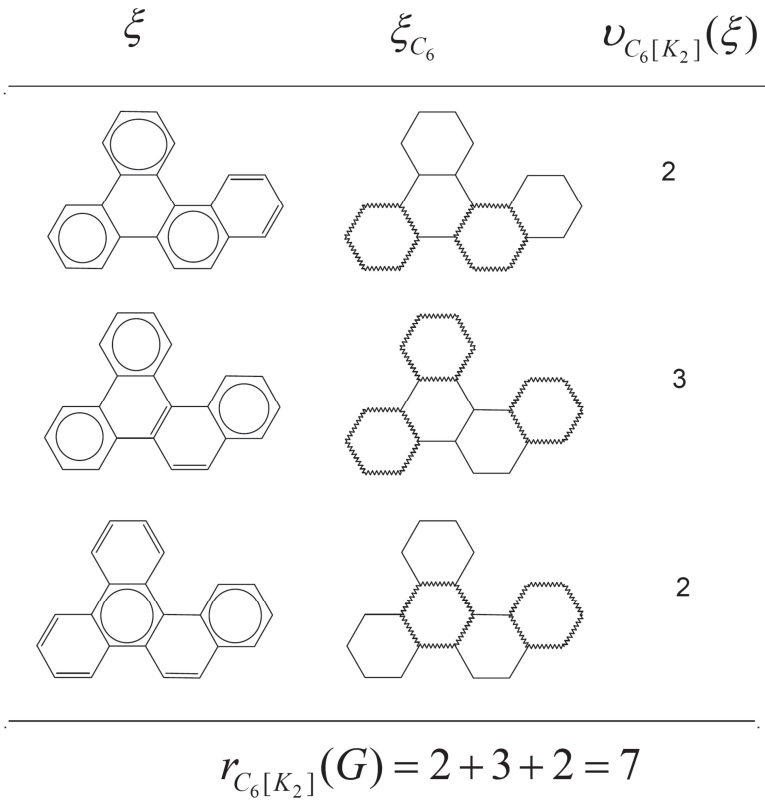

Figure 6. Three Clar structures as $C_{6} \mid K_{2}$-coverings for a benzo-chrysene, showing $C_{6}$-unique subgraphs, and their associated uniquenesses.

indicated as double). Also shown there is the consequent $K_{2}\left[K_{1}\right]$-uniqueness $v_{K_{2}\left[K_{1}\right]}(\xi)$. All other $K_{2}\left[K_{1}\right]$ coverings are symmetry equivalent to one of the 4 shown, whence we indicate also the number of members of such an equivalence class $\mathcal{O}(\xi)$. Finally the $K_{2}\left[K_{1}\right]$ richness is also given at the bottom of the figure.

Golumbic et $a l .{ }^{45}$ consider maximum matchings (i.e., of $K_{2}\left[K_{1}\right]$-coverings), and rather than the invariants $r_{K_{2}\left[K_{1}\right]}(G)$ or $\langle v\rangle_{K_{2}\left[K_{1}\right], G}$ consider the invariant which is the maximum value of $v_{K_{2}\left[K_{1}\right]}(\xi)$ amongst all the considered matchings. The $K_{2}$-unique subsets $\xi_{K_{2}}$ of a matching $\xi$ are (fully paired) Kekule structures in the induced subgraph $\xi_{K_{2}}{ }^{G}$, and of course $\xi_{K_{2}}$ is the sole Kekule structure of $\xi_{K_{2}}{ }^{G}$, which elsewhere ${ }^{59}$ then is called minimally Kekulenoid. Several known results ${ }^{45,46}$ for minimally Kekulenoid graphs then are relevant even chemically relevant (for HOMO-LUMO gaps of such graphs) - but this is not pursued here.

Another example involves Clar structures as $C_{6} \mid K_{2}$-coverings. For $G$ being the benzo-chrysene of Figure 6 , there are three $C_{6} \mid K_{2}$-coverings indicated there, and also this figure gives $C_{6}$-uniquenesses and $C_{6} \mid K_{2}$-richnesses.

Notably uniqueness is related to freedom (in a complementary fashion):

Proposition 4 - Let $\xi$ be an $A[B]$-covering of $G$, with forcing based on subgraphs of $A$-components of $\xi$, and let $\xi$ have freedom $\varphi_{A[B]}(\xi)$. Then $\varphi_{A[B]}(\xi)+v_{A[B]}(\xi)=|\xi|_{A}$.
Proof: For an $A[B]$-covering $\xi$, let $\zeta_{A}$ be a maximum-sized $A$-unique subgraph of $\xi$, and let the subgraph of the remaining $A$-components of $\xi$ be $\eta_{A}$, so that the full set of $A$ components of $\xi$ is $\zeta_{A} \oplus \eta_{A} \equiv \xi_{A}$ (where $\oplus$ indicates a union where the two combined sets are disjoint). Now within $\xi_{A}, \eta_{A}$ determines the complementary set of vertices (which is $V\left(\zeta_{A}\right)$ ) and so determines $\zeta_{A}$, whence $\eta_{A}$ forces $\zeta_{A}$ in $\xi_{A}{ }^{G}$. That is, $\varphi\left(\xi_{A}{ }^{G}\right)=\left|\eta_{A}\right|_{A}, v\left(\xi_{A}{ }^{G}\right) \leq\left|\zeta_{A}\right|_{A}$, and $\varphi\left(\xi_{A}{ }^{G}\right)+v\left(\xi_{A}{ }^{G}\right)=\left|\xi_{A}\right|_{A}$. But also the freedom for $\xi$ must be at least as large as that for the subgraph $\zeta_{A} \cup \eta_{A}$, so that $\varphi(\xi)+v(\xi) \geq|\xi|_{A}$. On the other hand, given a forcing set $\theta_{A}$ for $\xi$, the remnant subgraph $\rho_{A}$ of $A$-components of $\xi$ must be $A$-unique. That is, $|\theta|_{A}$ and $v(\xi) \leq\left|\rho_{A}\right|_{A}$, so that $\varphi(\xi)+v(\xi) \leq|\xi|_{A}$. Combination of the two inequalities on $|\xi|_{A}$ yields the desired result.

\section{"Functionalities" Related to Forcing}

A fundamental related graph invariant is found in the number $\#_{A[B]}(G)$ of $A[B]$-coverings of $G$. Another graph invariant is found in the force $f_{\mathcal{F}_{A[B]}}(G)$ of $G$, given as the sum of $\mathcal{F}$-forcing numbers over all $A[B]$ coverings. Two graphs $G_{1} \& G_{2}$ with disjoint vertex sets \& disjoint edge sets, give a direct sum $G_{1} \oplus G_{2}$ with vertex set $V\left(G_{1} \oplus G_{2}\right) \equiv V\left(G_{1}\right) \cup V\left(G_{2}\right) \quad \&$ edge set $E\left(G_{1} \oplus G_{2}\right) \equiv E\left(G_{1}\right) \cup E\left(G_{2}\right)$. Then

Proposition 5 - If $G$ is the direct sum of $G_{1} \& G_{2}$, then $\#_{A[B]}(G)$ is "multiplicative" in the sense that $\#_{A[B]}\left(G_{1} \oplus G_{2}\right)=\#_{A[B]}\left(G_{1}\right) \cdot \#_{A[B]}\left(G_{2}\right)$.

Proof: The disjointness of $G_{1} \& G_{2}$ implies an associated disjointness of the respective parts $\xi_{1} \subseteq G_{1}$ \& $\xi_{2} \subseteq G_{2}$ of an $A[B]$-covering $\xi$ of $G=G_{1} \oplus G_{2}$. Indeed the parts $\xi_{1} \& \xi_{2}$ are independent of one another, in the sense that $\xi_{1}$ must be an $A[B]$-covering of $G_{1} \&$ that $\xi_{2}$ similarly must be one for $G_{2}$. Thus the multiplicative result follows.

Proposition 6 - If $G$ is the direct sum of $G_{1} \& G_{2}$, then $f_{\mathcal{F}_{A(B)}}(G)$ is "derivative" in the sense that $f_{\mathcal{F}^{A[B]}}\left(G_{1} \oplus G_{2}\right)=f_{\mathcal{F} A[B]}\left(G_{1}\right) \cdot \#_{A[B]}\left(G_{2}\right)+$ $\#_{A[B]}\left(G_{1}\right) \cdot f_{\mathcal{F} A[B]}\left(G_{2}\right)$.

Proof: Following the notation of the preceding proof, one has

$$
f_{\mathcal{F} A[B]}\left(G_{1} \oplus G_{2}\right)=\sum_{\xi_{1}}^{G_{1}} \sum_{\xi_{2}}^{G_{2}} f_{\mathcal{F} A[B]}\left(\xi_{1} \oplus \xi_{2}\right)
$$

where the sums here are over $A[B]$-coverings of $G_{1} \&$ $G_{2}$, and $f_{\mathcal{F}_{A[B]}}(\xi)$ is understood to denote the $\mathcal{F}$ forcing number of the $A[B]$-covering $\xi$ of $G$. For a minimum $A[B]$-forcing set $\mathcal{S}$, each $S \in \mathcal{S}$ is a direct sum of $S_{1} \subseteq G_{1} \quad \& \quad S_{2} \subseteq G_{2}$, so that $f_{\mathcal{F} A[B]}\left(\xi_{1} \oplus \xi_{2}\right)=f_{\mathcal{F} A B]}\left(\xi_{1}\right)+f_{\mathcal{F} A[B]}\left(\xi_{2}\right)$. Then 


$$
\begin{aligned}
& f_{\mathcal{F} A(B)}(G)=\sum_{\xi_{1}}^{G_{1}} \sum_{\xi_{2}}^{G_{2}}\left\{f_{\mathcal{F} A[B]}\left(\xi_{1}\right)+f_{\mathcal{F} A[B]}\left(\xi_{2}\right)\right\}= \\
& \sum_{\xi_{1}}^{G_{1}} f_{\mathcal{F} A[B])}\left(\xi_{1}\right) \cdot\left\{\sum_{\xi_{2}}^{G_{2}} 1\right\}+\left\{\sum_{\xi_{1}}^{G_{1}} 1\right\} \cdot \sum_{\xi_{2}}^{G_{2}} f_{\mathcal{F} A[B]}\left(\xi_{2}\right)
\end{aligned}
$$

which then leads directly to the claim of the theorem.

If the notation for $f$ (or $f_{\mathcal{F}_{A[B]}}$ ) were here changed to $\partial \#$ (or $\partial_{\mathcal{F} A[B]} \#$ ), then the result of this proposition would appear even more like the standard Leibnizian relation. These results have already been noted $^{5}$ in the special context of the $e$-forcing of Kekule structures.

This result may be viewed as a qualitative "functionality" characterization of the forcing invariants for graphs. Of course, molecular graphs are not typically direct sums, but approximate versions of multiplicativity $\&$ derivativity should hold when $G$ nearly separates into separate vertex-disjoint pieces $G_{1} \& G_{2}$ - with the nearness to separation mediated by the smallness of the number of edges between the two prospective separate pieces. Moreover, different types of molecular properties may be viewed as at least approximately manifesting different types of such "functionality" dependences. Amongst common molecular properties $X$, several turn out to be at least approximately "additive", in the sense that they appear more like a sum of the invariants for their component parts (rather than a product, or derivative form). Indeed such expansions are complicit in classical chemistry, and there are monographs ${ }^{60-63}$ devoted just to such additive expansions for a single property. It can be argued that over all of chemistry such additive expansions are the most frequented type - though the decorations due to hetero-atoms (and consequent consideration of "colored" graphs) typically is an over-riding concern, which then tends to bring chemistry to the fore while often the graph theory is less noticed. Of the molecular properties, which one may argue are approximately "additive", one finds various system energies, magnetic susceptibilities, polarizabilities, molar refractivities, entropies, and various specific heats. Yet other properties may be argued to "scale" in a roughly additive manner - this including boiling points, heats of vaporization, chromatographic retention indices, some reactivities, and selected bioactivities. Thus it is of relevance that we have in hand the general result:

Proposition 7 - Let $X \& \partial X$ be graph invariants which respectively are "multiplicative" \& "derivative" in the sense that $X\left(G_{1} \oplus G_{2}\right)=X\left(G_{1}\right) \cdot X\left(G_{2}\right) \quad \&$ $\partial X\left(G_{1} \oplus G_{2}\right)=\partial X\left(G_{1}\right) \cdot X\left(G_{2}\right)+X\left(G_{1}\right) \cdot \partial X\left(G_{2}\right)$. Then the ratioed graph invariant $R(G) \equiv \partial X(G) / X(G)$ is "additive" in the sense that $R\left(G_{1} \oplus G_{2}\right)=R\left(G_{1}\right)+R\left(G_{2}\right)$.

The proof $^{5}$ is straight-forward. It may be noted that (other) derivative invariants have been successfully so ratioed to make additive invariants. See, e.g., Ref. 64. One widely utilized example, is found with the sum $\Sigma_{6}$ over all Kekule structures $\kappa$ of the number of 6-circuits appearing in each $\kappa$, whence one finds that $\Sigma_{6}$ is derivative (with respect to the associated multiplicative $K(G)$ ), and the ratio $\Sigma_{6} / K(G)$ is additive - and equal to the relevant ${ }^{26,27}$ net conjugated 6-circuit expectation $\sum_{C}^{6 \text {-cycle }}\langle C\rangle$. Still the most widely studied "additive" expansions are in terms of substructure counts (often described as "group-function" expansions, "substructural" expansions, etc). Another way to obtain an additive invariant is to take the logarithm of a (positive) multiplicative invariant. Thus it may be of some interest, that here with the various $\tau$-force ratios, we have another group of somewhat different additive invariants, in terms of which to make expansions for "additive" properties.

\section{"Full" Generalization of Forcing Idea}

Although a fair degree of generality was sought for the definition of forcing in the earlier sections, it seems to be even more general than so far indicated. Indeed G. Chartrand \& co-workers ${ }^{65-68}$ have explored yet other novel "forcing" constructions for graphs not covered in our preceding definitions. The "AIM Minimum Rank Special Graphs Work Group" has ${ }^{69}$ also considered a novel type of "forcing" resulting from a dynamic algorithmic process. Perhaps it was the difficulty in delimiting the full range of the idea of "forcing" which precluded Professor Harary from seeking to explain the idea in general.

Now a more far-ranging generalization beyond even the realm of graph theory is proposed. Let $\mathscr{P}$ be a parent set, with $\mathcal{C} \subseteq \mathscr{P}$ an (object) class, and $\mathscr{F}_{\mathcal{P}} \subseteq \mathscr{P}$ be a (forcing) family. Further let there be a partial ordering _ relation on $\mathscr{P}$, along with a commutative binary joining operation $\vee$ on $\mathscr{P}$ such that $S \vee T \neq \varnothing$ $\Rightarrow S{ }_{-} S \vee T \& T_{-} S \vee T$. Then $F \in \mathscr{F}_{\mathscr{P}}$ is a forcing of $S \in \mathcal{C}$ (with $F \_s$ ) if $F{ }_{-} \in \mathcal{C} \Rightarrow T=S$. An order-consistent norm $|P|$ on the elements $P \in \mathscr{P}$ is such that $P_{-} Q \Rightarrow|P| \leq|Q|$. Then the minimum value of $|F|$ for a forcing of $S \in \mathcal{C}$ is the freedom of $S$. The sum of the freedoms over all $S \in \mathcal{C}$ is the (net) force of $\mathcal{P}$.

For instance, $\mathscr{P}$ might be the subgraphs of a parent graph $G$, and $\mathcal{C}$ the class of $A[B]$-coverings, while $\mathscr{F}_{\mathscr{P}}$ is the family of subgraphs with all components isomorphic to $A$, and _ is the ordinary subgraph relation, with $S \vee T$ the union of $A$-components which are either identical or disjoint from one another in $S \& T$ ( and if not identical or disjoint, then $S \vee T \equiv \varnothing)$. Thence the ideas of $A[B]$-forcing emerge, as also do the ideas of freedom \& force when the order-consistent norm is taken to be the number of $A$-isomorphic components of a subgraph. If the relation _ is chosen as the comple- 
mentary relation $\subseteq_{c}$, then the (e)-forcing ideas for $K_{2}\left[K_{1}\right]$-covers emerge - and if chosen as the supergraph relation $\supseteq$, then the 6-forcing ideas for $C_{6}\left[K_{2}\right]$ coverings emerge. Similar emergences arise with similar choices except that $\mathcal{C}$ is the class of $A \mid B$ coverings.

But some graphic examples not yet explicated here would be nice. Recall that a subset of vertices of $G$ is called independent in $G$ if no two are adjacent in $G$, and a subset $D$ of vertices of $G$ is called dominant in $G$ if every vertex $\notin D$ is adjacent to one in $D$. Then one might choose $\mathscr{P}$ to be the dominant subgraphs of a parent graph $G$, and $\mathcal{C}$ the class of the minimum dominant subgraphs, while $\mathcal{F}_{\mathscr{P}}$ is the family of subgraphs with all components isomorphic to $K_{1}$, and ${ }_{-}$is the ordinary subgraph relation. Then one obtains the $\left\{K_{1}\right\}$ forcing proposed \& studied by Chartrand, Gavlas, Vandell, \& Harary. ${ }^{52}$ And their work on "forcing" has quite separately motivated some mathematical work, ${ }^{66-71}$ with it perhaps being worthwhile to explore the connections to what else described in the present article. Presumably the "forcing" idea may also be applied to minimal dominant sets, as well as to maximum $\&$ maximal independent sets. It may be noted that independence has been proposed ${ }^{72,73}$ to be of importance in identifying stabilities of fullerenes, and possibly other conjugated-carbon networks. These examples suggest that one imagine colored spanning subgraphs, with for example the independent set of vertices and its complement identifying the two different colors of vertices in what might be described as a $\left\{K_{1}, K_{1}^{\prime}\right\}[\varnothing]$-cover subject to an independence constraint for the vertices of the first color. One can even imagine allowing more than just two colors into such definitions, along with different constraints that might be imposed on such colored covers.

Even without coloring, more than two types of components of a covering can be entertained, e.g., for generalized Clar structures with components isomorphic to $C_{6}, K_{2}$, or $K_{1}$, with constraints that there are not two $K_{1}$ components at adjacent sites and no three $K_{2}$ components around a 6-cycle of $G$. Such (maximal) $C_{6}\left|K_{2}\right| K_{1}$-coverings are relevant ${ }^{74}$ in circumstances when (generalized) Clar structures with unpaired sites are relevant -i.e., for the cases of radicaloid systems as are especially relevant in the consideration of magnetic \& electrical properties of benzenoid polymers \& graphene.

Finally it may be noted that our ultimate general idea of forcing has many points in common with the general notion of "filters" from topology. See, Cartan's original work ${ }^{75}$ or for a more general view Bourbaki. ${ }^{76}$ But we do not pursue this here.

Notably in all the so imagined circumstances in the earlier sections, the "derivativity" result of the preceding section seems to hold.

\section{CONCLUSION}

The overall idea of forcing (e.g., for various sorts of graph covers) seems to be of great generality, going much beyond the Kekulean case earlier considered just as Professor Harary intimated in 1990. Forcing is here presented in an ultimately far-reaching generality, to reveal it as mathematically fundamental, with a few relevant characteristics developed. There are numerous cases where forcing seems to have significant chemical suggestiveness, especially in the context of valencebond-theoretic ideas, and (closely related) Clar-theoretic ideas. That is, forcing measures how "non-rigid" valence-bond structures are, or alternatively, how much "freedom" they have; and there is an associated implication, that given sufficient rigidity such a structure is determined in terms of a few local pieces - or alternatively that the structure has a high degree of "uniqueness", which is also here formally defined. Should all Kekule structures share a common mode of rigidity, this would implicate a "long-range order", discussed in general in Refs. 77,78; and with different physical consequences - as in Refs. 38-40,62,79-81,38. On the other hand, from a molecular-orbital perspective, there are relevant spanning subgraphs (identified with Sachs subgraphs, ${ }^{23}$ supplemented with single-site components to make the result spanning), for which forcing ideas might somehow also be relevant. Further with the consequent novel force invariants for graphs, there are possible uses in QSPR \& QSAR. The relevance of "additivity" (say for QSPR \& QSAR) and how to obtain this behavior from the manifested "derivativity" of typical force invariants has been clarified. The use of different types of "incomplete" coverings (or alternatively complete coverings with many $K_{1}$ components) occurs when one looks at the problem ${ }^{82}$ of bidentate ligands attached to a molecular skeleton. Also this arises in absorption of dimers on surfaces where it is ${ }^{83,84}$ natural to also use a variable weight for the lone $\left(K_{1}\right)$ sites - and this presumably might lead to development of forcing ideas for weighted coverings, as in fact should also be relevant in a resonance theoretic circumstance. In the use of coverings to represent isomers with bidentate (or even higher multidentate) ligands, the equivalence classes of symmetry equivalent substitutional patterns becomes relevant, and undoubtedly leads to further mathematical novelties. Both the full utility and even the full generality of forcing remain to be delineated and explored.

Acknowledgements. The support of the Welch Foundation of Houston, Texas, is acknowledged (through grant BD-0894). Further, stimulating discussions both with Milan Randić and with the late Professor Frank Harary are acknowledged, as well as some helpful comments from the two referees. 
This paper is dedicated to Frank, and to his memory.

\section{REFERENCES}

1. F. Harary, The Theory of Graphs, John Wiley \& Sons, NY, 1970.

2. F. Harary, Chemical Applications of Graph Theory, pp. 5-10, ed. A. T. Balaban, Academic Press, NY, 1975.

3. J. Math. Chem. 4 (1990), special editors D. J. Klein and M. Randić.

4. M. Randić and D. J. Klein in Mathematical and Computational Concepts in Chemistry, pp. 274-282, ed. N. Trinajstić, Ellis Horwood Pub., Chichester, England, 1986.

5. D. J. Klein and M. Randić, J. Comp. Chem. 8 (1987) 516-521.

6. F. Harary, D. J. Klein, and T. P. Živković, J. Math. Chem. 6 (1991) 295-306.

7. F. Zhang and X. Li, Disc. Math. 140 (1995) 253-263.

8. L. Pachter and P. Kim, Disc. Math 190 (1998) 287-294.

9. F. Lam and L. Pachter, Theor. Comp. Sci. 303 (2003) 409-416.

10. M. E. Riddle, Disc. Math. 245 (2002) 283-292.

11. D. Vukičević and J. Sedlar, Math. Commun. 9 (2004) 169-179.

12. P. Afshani, H. Hatami, and E. S. Mahmoodian, Aust. J. Comb. 30 (2004) 147-160.

13. P. Adams, M. Mahdian, and E. S. Mahmoodian, Disc. Math. 281 (1994) 33-43.

14. S. Kleinerman, Disc. Math. 306 (2006) 66-73.

15. H. Wang, Lec. Notes Comp. Sci. 5573 (2009) 258-268.

16. H. Zhang, D. Ye, and W. C. Shiu, Disc. Appl. Math. 158 (2010) 573-582.

17. X. Jiang and H. Zhang, Disc. Appl. Math. 159 (2011) 15811593.

18. J. Sedlar, Disc. Appl. Math. 160 (2012) 2306-2313.

19. Z. Che and Z. Chen, Commun. Math. and Comp. Chem. (MatCh) 56 (2006) 849-868.

20. T. Doslić, J. Math. Chem. 41 (2007) 217-229.

21. N. Trinajstić and D. Vukicević, Struct. Chem. 18 (2007) $807-$ 812.

22. D. Vukičević and N. Trinajstić, J. Math. Chem. 42 (2007) $575-$ 583.

23. H. Wang, D. Ye, and H. Zhang, J. Math. Chem. 43 (2008) 457475.

24. Z. Che and Z Chen, Commun. Math. Comp. Chem. (MatCh) 66 (2011) 93-136.

25. J. Cai and H. Zhang, Commun. Math. Comp. Chem. (MatCh) 67 (2012) 289-312.

26. Z. Che and Z. Chen, Commun. Math. Comp. Chem. (MatCh) 69 (2013) 721-132.

27. L. Xu, H. Boam and F. Zhang, Commun. Math. Comp. Chem. (MatCh) 70 (2013) 493-500.

28. H. Deng, Commun. Math. Comp. Chem. (MatCh) 60 (2008) 183192.

29. H. Deng, Commun. Math. Comp. Chem. (MATCH) 58 (2007) 675-682.

30. H. Zhang, D. Ye, and W. C. Shiu, Commun. Math. Comp. Chem. (MatCh) 158 (2010) 573-582.

31. Q. Zhang, H. Bian, and E. Vumar, Commun. Math. Comp. Chem. (MatCh) 65 (2011) 799-806.

32. Q. Yang, D. Ye, H. Zhang, and Y. Lin, Commun. Math. Comp. Chem. (MatCh) 67 (2011) 281-288.

33. J. Cai and H. Zhang, Commun. Math. Comp. Chem. (MatCh) 69 (2013) 733-754.

34. N. Trinajstić, Chemical Graph Theory (CRC Press, Boca Raton, 1982-1991)

35. E.g., sec. 7-5 of the $19603^{\text {rd }}$ edn. of L. Pauling, The Nature of the Chemical Bond (Cornell U. Press, Ithaca)

36. L. Pauling, Acta Cryst. B 36 (1980) 1898.
37. W. C. Herndon, Thermochimica Acta 8 (1974) 225-237.

38. W. C. Herndon and M. L. Ellzey Jr., J. Am. Chem. Soc. 96 (1974) 6631-6642

39. M. Randić, Tetrahedron 33 (1977) 1905-1920.

40. M. Randić, J. Am. Chem. Soc. 99 (1977) 444-450.

41. J. A. N. F. Gomes and R. B. Mallion, Rev. Port. Quim. 21 (1979) 82-89.

42. D. J. Klein and N. Trinajstić, Pure Appl. Chem. 61 (1989) $2107-$ 2115 .

43. T. K. Dickens, J. A. N. F. Gomes, and R. B. Mallion, J. Chem. Th. Comp. 7 (2010) 3661-3674.

44. M. Randić, Chem. Rev. 103 (2002) 3449-3605.

45. S. El-Basil and M. Randić, J. Chem. Soc. Faraday Trans. 284 (1988) 1875

46. I. Gutman, I. Naturforsch. 36 (1981) 69.

47. M. Randić, J. Mol. Struc. (Theochem) 229 (1991) 139.

48. S. El-Basil and I. Gutman, I. Chem. Phys. Lett. 94 (1983) 188.

49. J. R. Dias, J. Mol. Struct. (Theochem) 581 (2002) 59.

50. D. J. Klein and L. Bytautas, J. Phys. Chem. A 103 (1999) 51965210

51. O. Ivanciuc, L. Bytautas, and D. J. Klein, J. Chem. Phys. 116 (2002) 4736-4748

52. O. Ivanciuc, D. J. Klein, and L. Bytautas, Carbon 40 (2002) 2063-2083.

53. D. J. Klein, Pure Appl. Chem. 80 (2008) 1399-1414.

54. R. S. Nord, J. Math. Chem. 9 (1992) 39-53.

55. J. Dayantis, Comp. Theor. Polymer Sci. 10 (2000) 507-522.

56. E. Clar, The Aromatic Sextet (John Wiley \& Sons, NY, 1970)

57. D. J. Klein, Commun. Math. Comp. Chem. (MatCh) 42 (2000) 7 21.

58. M. C. Golumbic, T. Hirst, and M. Lewenstein, Algorithmica 31 (2001) 139-154

59. D. J. Klein and A. Misra, Croat. Chem. Acta 77 (2004) 179-191.

60. V. M. Tatevskii, V. A. Benderskii, and S. S. Yarovoi, Rules and Methods for Calculating the Physico-Chemical Properties of Paraffinic Hydrocarbons (English translation from Pergamon Press, Oxford, 1961).

61. J. G. Janz, Estimation of Thermodynamic Properties of Organic Compounds (John Wiley, NY, 1968).

62. J. D. Cox and G. Pilcher, Thermochemistry of Organic and Organometallic Compounds (Academic Press, NY, 1970).

63. S. W. Benson, Thermochemical Kinetics (John Wiley \& Sons, NY, 1976).

64. D. J. Klein, Intl. J. Quantum Chem. S 20 (1986) 153-171.

65. G. Chartrand, H. Gavlas, R. C. Vandell, and F. Harary, J. Comb. Math. Comb. Comput. 25 (1997) 161-174.

66. G. Chartrand and P. Zhang, Discuss. Math. Graph Theory 19 (1999) 45-58.

67. G. Chartrand and P. Zhang, Math. Bohem. 126 (2001) 711-720.

68. G. Chartrand and P. Zhang, Czech. Math. J. 51 (2001) 847-858.

69. AIM Minimum Rank - Special Graphs Work Group, Lin. Alg. \& Its Appl. 428 (2008) 1628-2648.

70. L-D. Tong, Disc. Appl. Math. 157 (2009) 1159-1163.

71. L-D. Tong, Disc. Math. 309 (2009) 1623-1628.

72. S. Fajtlowicz, P. W. Fowler, H. Hansen, and K. M. Rogers, J. Chem. Soc. Perkin Trans. 2 (1998) 1531-1534.

73. S. Fajtlowicz and C. E. Larson, Chem. Phys. Lett. 377 (2003) 485-490.

74. A. Misra, T. G. Schmalz, and D. J. Klein, J. Chem. Inf. Model. 49 (2009) 2670-2676

75. H. Cartan, Compt. Rend. Acad. Paris 205 (1937) 595-598.

76. N. Bourbaki, chapters 1 and 2 of General Topology (AddisonWesley Pub., Reading, MA, 1970).

77. D. J. Klein, Intl. J. Quantum Chem. S 13 (1979) 293-303.

78. D. J. Klein, T. P. Živković, and R. Valenti, Phys. Rev. B 43 (1991) 723-727.

79. D. J. Klein, T. G. Schmalz, W. A. Seitz, and G. E. Hite, Intl. J. 
Quantum Chem. S 19 (1986) 707-718.

80. D. J. Klein, T. G. Schmalz, M. A. Garcia-Bach, R. Valenti, and T. P. Živković, Phys. Rev. B 43 (1991) 719-722.

81. M. A. Garcia-Bach, A. Penaranda, and D. J. Klein, Phys. Rev. B 45 (1992) 10891-10901.
82. D. J. Klein and A. H. Cowley, J. Am. Chem. Soc. 100 (1978) 2593-2599.

83. O. J. Heilmann and E. H. Lieb, Commun. Math. Phys. 25 (1972) 190-232.

84. A. J. Phares, J. Math. Phys. 25 (1984) 1756. 